\title{
Design of a Novel High Power V-Band Helix-Folded Waveguide Cascaded Traveling Wave Tube Amplifier
}

\author{
Tianxiang Zhuge and Yulu Hu \\ School of Physical Electronics, University of Electronic Science and Technology of China, Chengdu 610054, China \\ Correspondence should be addressed to Yulu Hu; yuluhu@uestc.edu.cn
}

Received 24 March 2015; Revised 12 May 2015; Accepted 17 May 2015

Academic Editor: Gianluca Traversi

Copyright (C) 2015 T. Zhuge and Y. Hu. This is an open access article distributed under the Creative Commons Attribution License, which permits unrestricted use, distribution, and reproduction in any medium, provided the original work is properly cited.

\begin{abstract}
A design of a V-band Helix-Folded Waveguide (H-FWG) cascaded traveling wave tube (TWT) is presented. In this cascaded structure, a digitized nonlinear theory model is put forward first to simulate these two types of the tubes by common process. Then, an initial design principle is proposed, which can design these two different kinds of tubes universally. Using this principle, a high-gain helix TWT is carefully designed as a first stage amplifier followed by a FWG TWT to obtain high power. Simulations predict that a peak power of $800 \mathrm{~W}$ with saturated gain of $60 \mathrm{~dB}$ from $55 \mathrm{GHz}$ to $60 \mathrm{GHz}$ can be achieved.
\end{abstract}

\section{Introduction}

The V-band frequency range $(50-75 \mathrm{GHz})$ is a region of the millimeter wave spectrum with great potential application for intersatellite communications and high-performance radar applications, including atmospheric studies, space debris detection, precise tracking, and high resolution imaging [13]. However, it has to face to the size and power limitation at this frequency range, when using traditional helical traveling wave tube $[1,4]$ and some novel structure TWTs [5-7] on such high-frequency band. However, some metal structure TWTs (Coupled Cavity (CC) TWT [8], FWG TWT [9, 10], and so on) can obtain high power but low gains in single tube for strong backward wave oscillation (BWO) instability, also for some FWG cascaded TWTs [11, 12] and CC-FWG cascaded TWTs [13].

In order to obtain a high power and high-gain V-band traveling wave tube in feasible manufacture way, we designed a novel H-FWG cascaded traveling wave tube amplifier. In this design, we use helical traveling wave tube as a first stage amplifier, which is famous for its wide bandwidth and high gain and then exported the amplified signal into the FWG traveling wave tube to get high power. The FWG traveling wave tube has large power capability for its full metal structure and is chosen to get high power in the V-band frequency range.
A digitized beam-wave interaction theory model [14, $15]$ is used to analyze the performance of the designed helix-FWG cascaded traveling wave tube, which has been developed and included in MTSS [16]. In this model the digitized fields, getting the data from electromagnetic (EM) simulation software for arbitrary SWSs, are used to interact with the beam keeping the energy translation and conservation between the beam and the EM wave. Due to its general way to deal with the beam-wave interaction process, this digitized theory model can be used to simulate and analyze the nonlinear performance of traveling wave tube with different SWS.

A helix-FWG cascaded traveling wave tube testified the design method and is optimized. Finally, the simulation of the saturated output power is above $34 \mathrm{~W}$ with the saturated gain above $33 \mathrm{~dB}$ in $5 \mathrm{GHz}$ bandwidth in the first stage helical TWT. And, for the cascaded FWG traveling wave tube in same bandwidth, the saturated gain is above $18 \mathrm{~dB}$ achieving the output power to be $800 \mathrm{~W}$. After matching the inputoutput power in the connection, the total cascaded tube achieves $60 \mathrm{~dB}$ gain and $800 \mathrm{~W}$ in $5 \mathrm{GHz}$ band. The illustration of the helix-FWG cascaded TWT model is presented in Figure 1.

The rest of the paper is organized as follows. Section 2 presents the sketch of the digitized nonlinear beam-wave interaction theory model, and Section 3 introduces the 


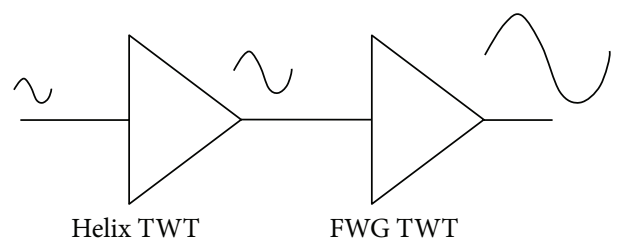

FIGURE 1: (Black and white) H-FWG cascaded TWT model.

principle of the TWT design. Based on the design principle and the digitized theory model, the design and analysis of the H-FWG cascaded TWT are detailed in Section 4. A conclusion is followed at the end of the paper.

\section{The Digitized Nonlinear Theory Model [14]}

The digitized nonlinear theory model is a quasi-3D nonlinear theory model developed and included in MTSS [16]. It gets electromagnetic field distribution of arbitrary SWSs from the numerical simulation software such as HFSS, CST, and HFCS and then uses the energy conservation principle to get the energy exchange between the beam and the EM wave. Consequently, this digitized theory model is allowed to analyze the nonlinear performance of many kinds of traveling wave tube using different periodic high-frequency structures.

In the digitized nonlinear theory, RF fields acting on the electron beam propagating in a SWS are represented as

$$
\begin{aligned}
\mathbf{E}_{\mathrm{rf}}(\mathbf{x}, t) & =\sum_{n} \mathbf{E}_{\mathrm{rf}, n}(\mathbf{x}, t)=\sum_{n}\left[f_{n}(z) \mathbf{e}_{n}(\mathbf{x}) e^{-i \omega_{n} t}\right], \\
\mathbf{H}_{\mathrm{rf}}(\mathbf{x}, t) & =\sum_{n} \mathbf{H}_{\mathrm{rf}, n}(\mathbf{x}, t)=\sum_{n}\left[f_{n}(z) \mathbf{h}_{n}(\mathbf{x}) e^{-i \omega_{n} t}\right] .
\end{aligned}
$$

The RF electric field $\mathbf{E}_{\mathrm{rf}}$ and magnetic field $\mathbf{H}_{\mathrm{rf}}$ are composed of many different time harmonics, whose angular frequency is $\omega_{n}$ and complex amplitude varying along the $z$ axial is $f_{n}(z)$. Subscript $n$ indicates the harmonic number and $\mathbf{e}_{n}(\mathbf{x})$ and $\mathbf{h}_{n}(\mathbf{x})$ are the RF field profiles without electron beam loaded, which will be obtained by EM simulation of HFSS [17], HFCS [16], CST [18], and so forth. Once obtaining the RF field profiles, the evolution of the RF field amplitude can be developed based on the law of energy conservation, and finally we get

$$
\begin{aligned}
& {\left[\frac{\partial}{\partial z}+\alpha_{n}(z)\right] f_{n}(z)=-\frac{1}{2} \int_{z}^{z+\lambda_{h}} \frac{d z}{\lambda_{h}} \int_{t}^{t+T} \frac{d t}{T}} \\
& \cdot \iint_{A} \mathbf{j} \cdot \mathbf{e}_{n}^{*}\left(\mathbf{x}_{\perp}, z_{0}\right) e^{-i m \varphi_{n}} e^{i \omega_{n} t} d s, \quad z_{0} \in\left[0, \lambda_{h}\right) .
\end{aligned}
$$

Here $\alpha_{n}$ means the attenuation constant, $\lambda_{h}$ is the axial period length of the structure, and $T$ is the period time. $\mathbf{j}=\rho \mathbf{v}$ is the beam current density. $m$ indicates the number of the period among which $z$ is solving. And $\varphi_{n}$ is the phase shift for the $n$th RF signal in one period.

For different type of slow-wave structure (SWS), $\mathbf{e}_{n}(\mathbf{x})$ and $\mathbf{h}_{n}(\mathbf{x})$ are quite different. Without care for the analytic model of the slow-wave structure, we can use (1) to get the EM field and then to push the particles in the beam. The beam current then affects the field amplitude $f_{n}(z)$, which will inevitably change the status of the beam and thus the current. Following this circulation, we can trace the process of the beam-wave interaction and get the nonlinear performance of the TWT.

\section{Design Principle of TWT}

As a first step, to get the desired output power and gain, the beam voltage and the beam density in the tunnel (FWG or helix) in beam-wave interaction region have to be carefully selected, which are of the most important factors limiting the output power and gain. As it is well known, the output power $P_{o}$ can be estimated by beam voltage $V$, beam current $I_{0}$, and electrical efficiency $\eta$; that is,

$$
P_{o}=\eta V I_{0}
$$

From (3), we get beam current:

$$
I_{0}=\frac{P_{o}}{\eta V} .
$$

And the beam density in the tunnel (FWG or helix) in beamwave interaction region is

$$
J=\frac{I_{0}}{\pi b^{2}}=\frac{I_{0}}{\pi\left(\rho r_{a}\right)^{2}}=\frac{P_{o}}{\pi\left(\rho r_{a}\right)^{2} \eta V} .
$$

Here $b$ is the beam radius, $\rho$ is the beam filling ratio, and $r_{a}$ is the radius of the tunnel (FWG or helix). On the other hand, the relativity factor $\gamma\left(=1 / \sqrt{1-v_{0}^{2} / c^{2}}\right)$ can be estimated by

$$
\gamma-1=\frac{e}{m} \frac{V}{c^{2}}
$$

where $e / m$ is the electron charge to mass ratio and $c$ is speed of light, from which we get the initial beam speed $v_{0}$ :

$$
v_{0}=\sqrt{\frac{e}{m}\left(\frac{\gamma+1}{\gamma^{2}}\right) V} .
$$

Define a slow-varying and dimensionless parameter $\Gamma$ as

$$
\Gamma=\frac{\omega}{v_{p}} r_{a} \approx \frac{\omega}{v_{0}} r_{a}
$$

And combining (7) and (8), the tunnel radius $r_{a}$ can be presented as

$$
r_{a}=\frac{\Gamma}{\omega} \sqrt{\frac{e}{m}\left(\frac{\gamma+1}{\gamma^{2}}\right) V} .
$$

Inserting (9) into (5), the beam density can finally be written as

$$
J=\frac{m P_{\mathrm{o}}}{e \pi \eta(\gamma+1)}\left(\frac{\gamma \omega}{\rho \Gamma V}\right)^{2} .
$$




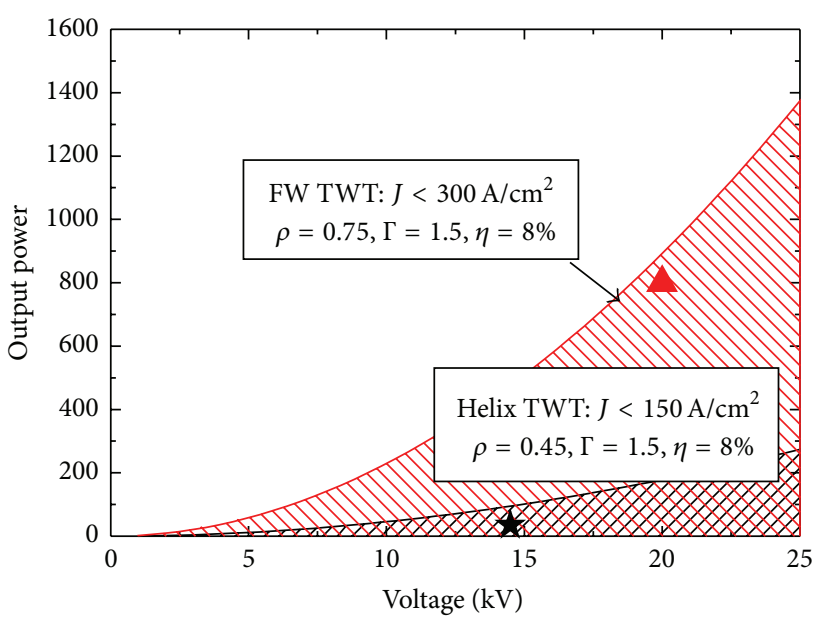

(a)

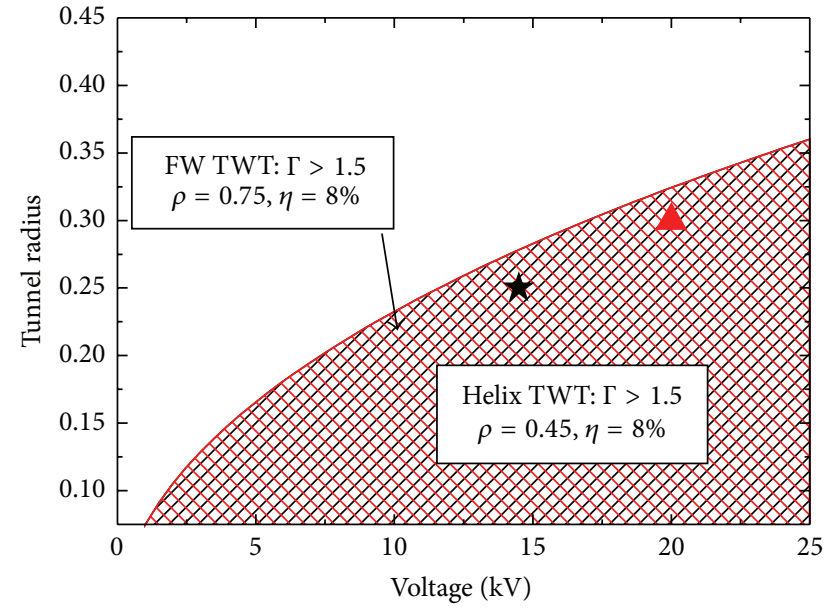

(b)

FIGURE 2: (a) Relationship between output power and voltage for helix TWT (black slashed region) and FWG TWT (red backslash region), which are partially overlapped. (b) Relationship between tunnel radius and voltage for helix TWT (black slash region) and FWG TWT (red backslash region), which are completely overlapped.

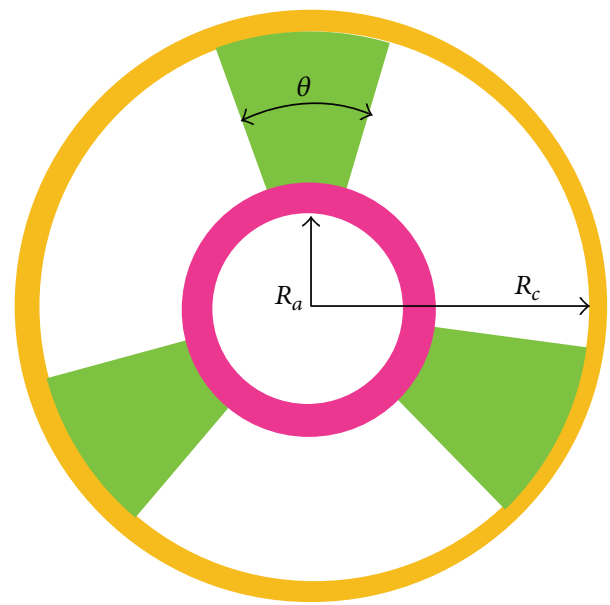

FIGURE 3: Illustration of the cross section of the helix SWS.

And the Brillouin magnet field will be

$$
\begin{aligned}
B_{B} & =\frac{1}{b}\left(\frac{2 m I}{\pi e \varepsilon_{0} v_{0}}\right)^{1 / 2} \\
& =\left[\frac{2 J}{\varepsilon_{0} \sqrt{(e / m)^{3}\left((\gamma+1) / \gamma^{2}\right) V}}\right]^{1 / 2} \\
& \approx 17.5 \times \frac{(\gamma J)^{1 / 2}}{[(\gamma+1) V]^{1 / 4}}(\mathrm{Gs}) .
\end{aligned}
$$

Based on the current density limitation of certain kind of TWT, (10) and (9), we can get the relationship between voltage and output power as well as the tunnel radius and voltage. After choosing proper electronic efficiency, beam filling ratio, and parameter $\Gamma$, a proper voltage and the tunnel radius can be selected to meet our design.

\section{Design of the H-FWG Cascaded Traveling Wave Tube}

In the design of H-FWG cascaded traveling wave tube, we first select a proper voltage and tunnel radius and then optimize the SWS and beam-wave interaction profile of the helix TWT and the FWG TWT separately. Cascading these two sections, an output power of $800 \mathrm{~W}$ and a gain of $55 \mathrm{~dB}$ are expected in the V-band of $55 \mathrm{GHz}$ to $60 \mathrm{GHz}$.

4.1. Choice of Voltage. In this V-band cascaded TWT design, we first set a proper parameter $\Gamma$ and electronic efficiency $\eta$ (here we set $\eta=8 \%$ and $\Gamma=1.5$ as initial value). The beam density of helix TWT is chosen less than $150 \mathrm{~A} / \mathrm{cm}^{2}$ and the filling ratio is set to be 0.45 for helix TWT. For FWG TWT with greater power capacity, the beam density is chosen as $300 \mathrm{~A} / \mathrm{cm}^{2}$ and the filling ratio is 0.75 . Using (9) and (10), we get the relationship between output power and voltage, as well as the tunnel radius and voltage, as shown in Figure 2.

After careful optimization, a voltage of $14.5 \mathrm{KV}$ is selected for the first stage helix TWT, whose corresponding output power is $34 \mathrm{~W}$ and helix radius is $0.25 \mathrm{~mm}$, shown as the black star in Figure 2. For the FWG TWT, the voltage is set to $20 \mathrm{KV}$, corresponding to an output power of $800 \mathrm{~W}$ and tunnel radius of $0.3 \mathrm{~mm}$, demonstrated as the red triangle in Figure 2. And the current is to be $32.5 \mathrm{~mA}$ for helix TWT and $450 \mathrm{~mA}$ for FWG TWT.

4.2. Design of Helix TWT. A simple helix SWS with three APBN sector support rods is selected (shown in Figure 3), which can be manufactured easily. The design principle of the other SWS parameters, except fixed helix radius to $0.25 \mathrm{~mm}$, 


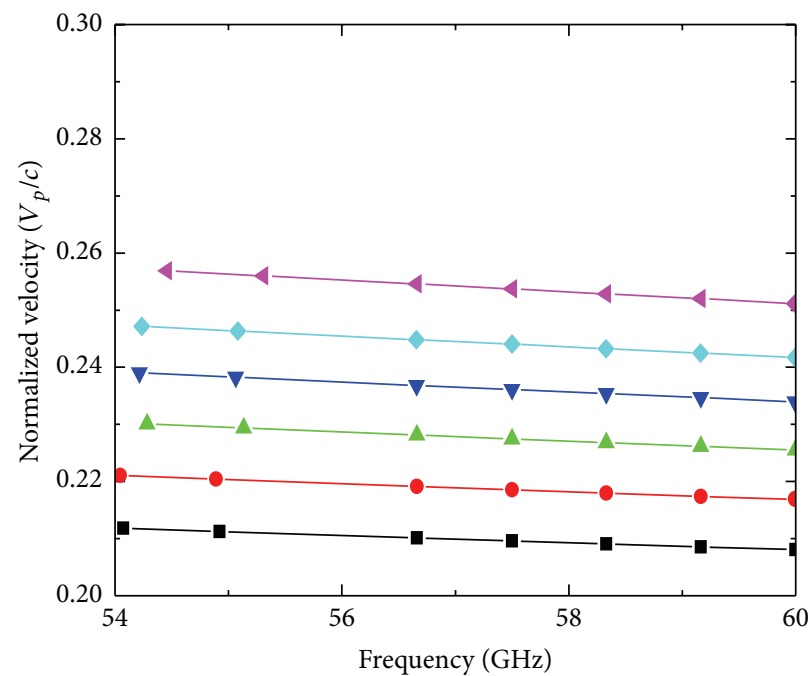

(a)

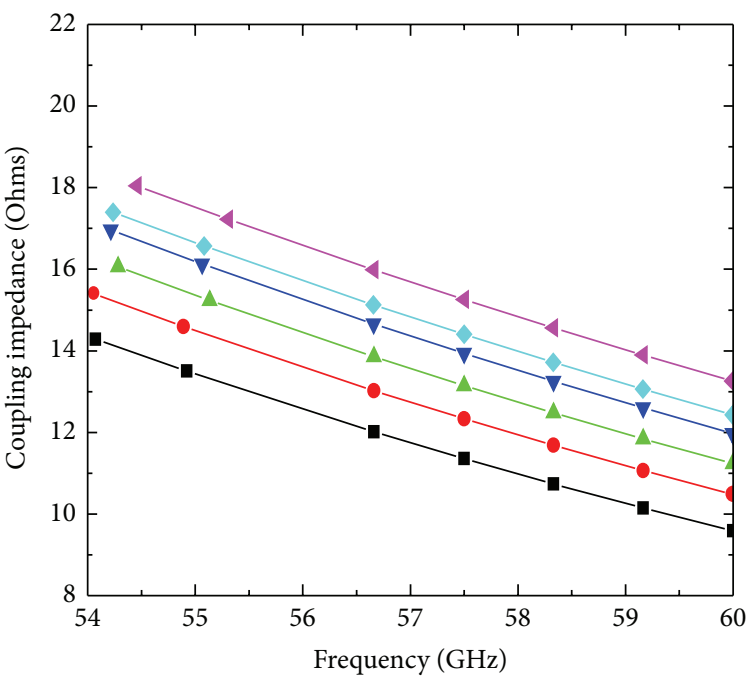

(b)

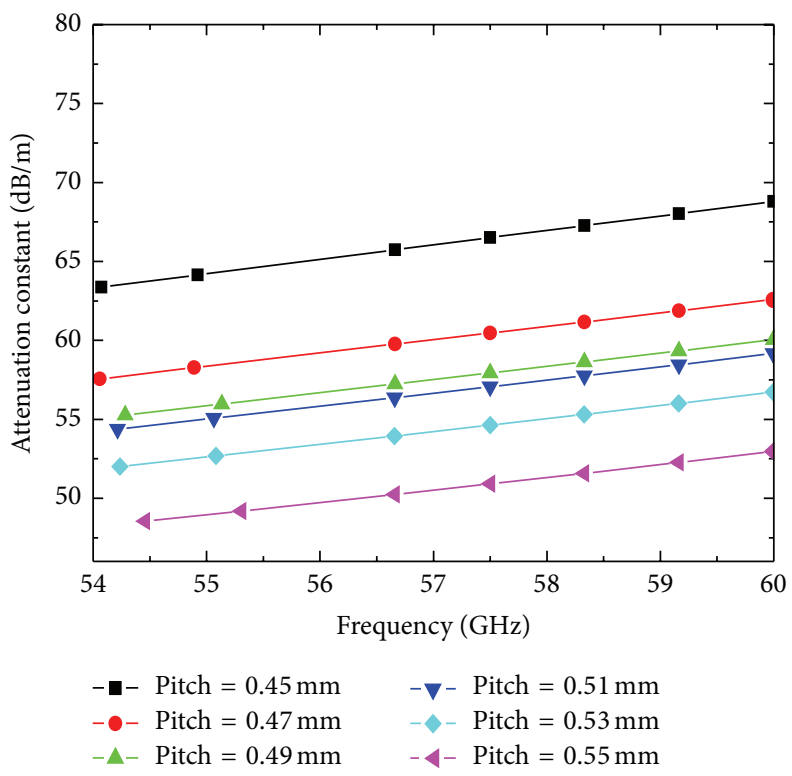

(c)

FIGURE 4: (a) Dispersion, (b) coupling impedance, and (c) attenuation constant for the helix SWS with pitch ranging from $0.45 \mathrm{~mm}$ to $0.55 \mathrm{~mm}$.

is seeking the suitable dimensions to maximize the coupling impedance and to obtain flat dispersion between $55 \mathrm{GHz}$ and $60 \mathrm{GHz}$. After careful optimization, the helix SWS structure parameters are shown in Table 1 . The helix pitch ranges from $0.45 \mathrm{~mm}$ to $0.55 \mathrm{~mm}$. For later beam-wave interaction optimization, the helix SWS with different helix pitch will be simulated with a step of $0.02 \mathrm{~mm}$.

The normalized velocity, coupling impedance, and attenuation constant for the helix SWS with helix pitch ranging from $0.45 \mathrm{~mm}$ to $0.55 \mathrm{~mm}$ are shown in Figure 4 .

It is simulated and optimized of the beam-wave interaction with pitch and attenuation profile (shown in Figure 5), using the simulation code developed on the model discussed in Section 2. Also BWO is considered by using an additional attenuator in input circuit and pitch step in output circuit. The pitch of the circuit is finally set to be $0.505 \mathrm{~mm}$ and then step

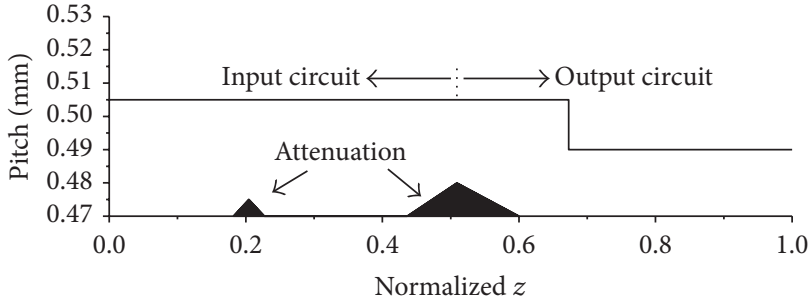

FIGURE 5: Pitch and attenuation profiles in helix TWT (black and white).

to $0.49 \mathrm{~mm}$, respectively. The profile of output power and gain along $z$-axis at $57 \mathrm{GHz}$ is shown in Figures 6(a) and 6(b). And the saturated output power and saturated gain in band are shown in Figure 6(c). Obviously, the output power of the first 


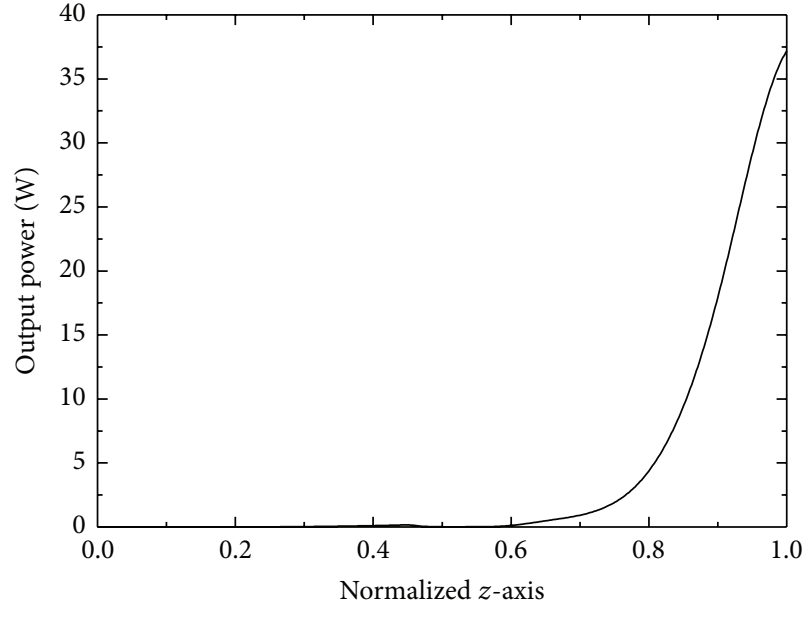

$-57 \mathrm{GHz}$

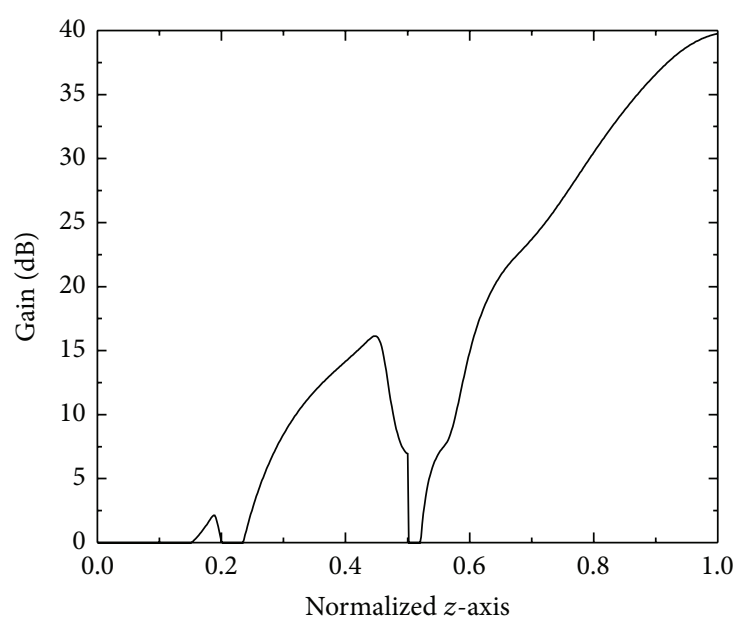

— $57 \mathrm{GHz}$ gain

(a)

(b)

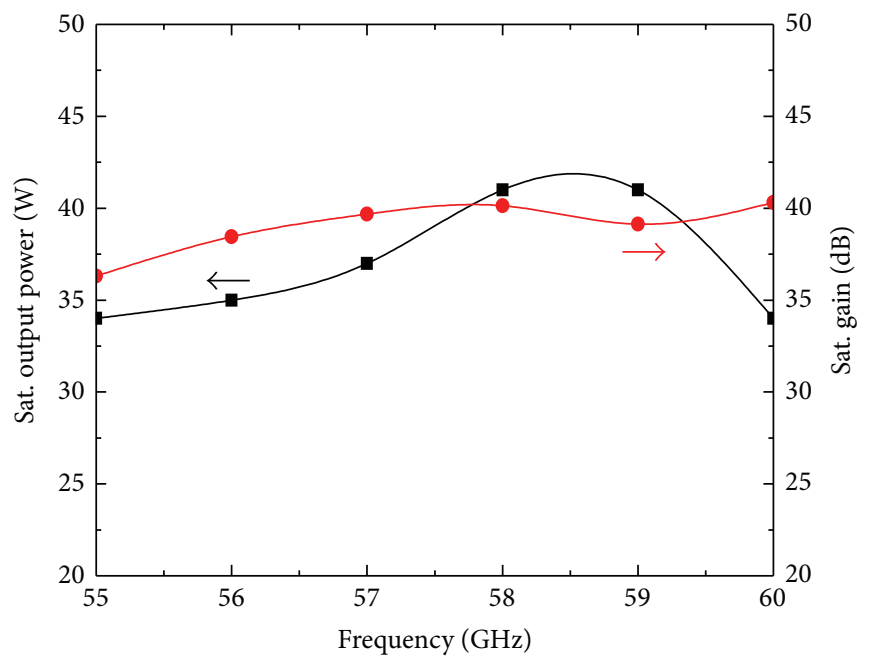

$\rightarrow$ Sat. output power

Sat. gain

(c)

FiguRE 6: (a) Output power versus $z$ at $57 \mathrm{GHz}$, (b) gain versus $z$ at $57 \mathrm{GHz}$, and (c) saturated output power and saturated gain ranging from $55 \mathrm{GHz}$ to $60 \mathrm{GHz}$.

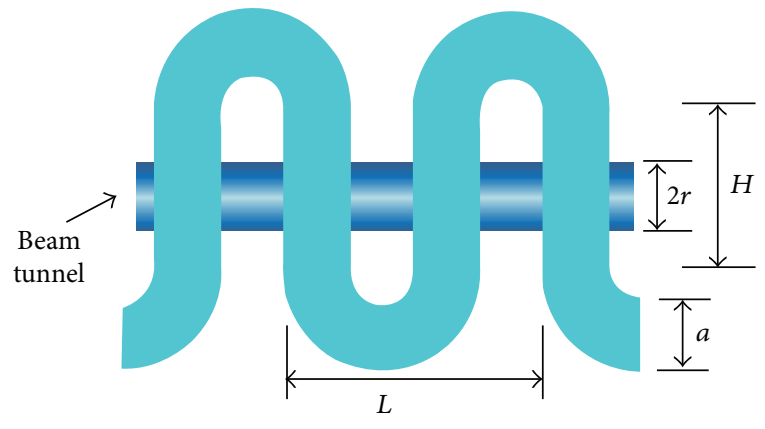

(a)

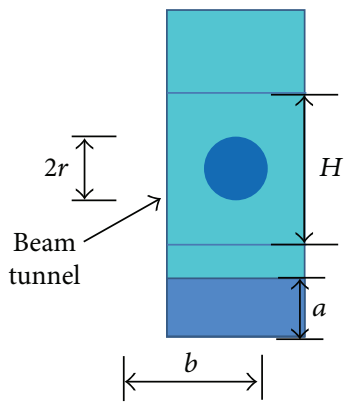

(b)

FIGURE 7: Illustration of (a) side view and (b) front view of the FWG SWS. 


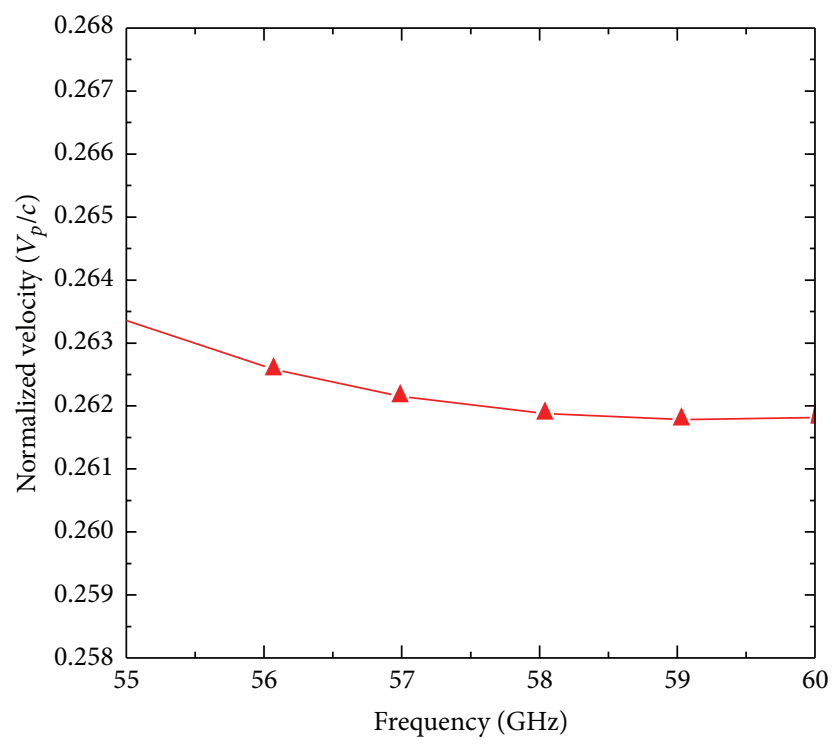

(a)

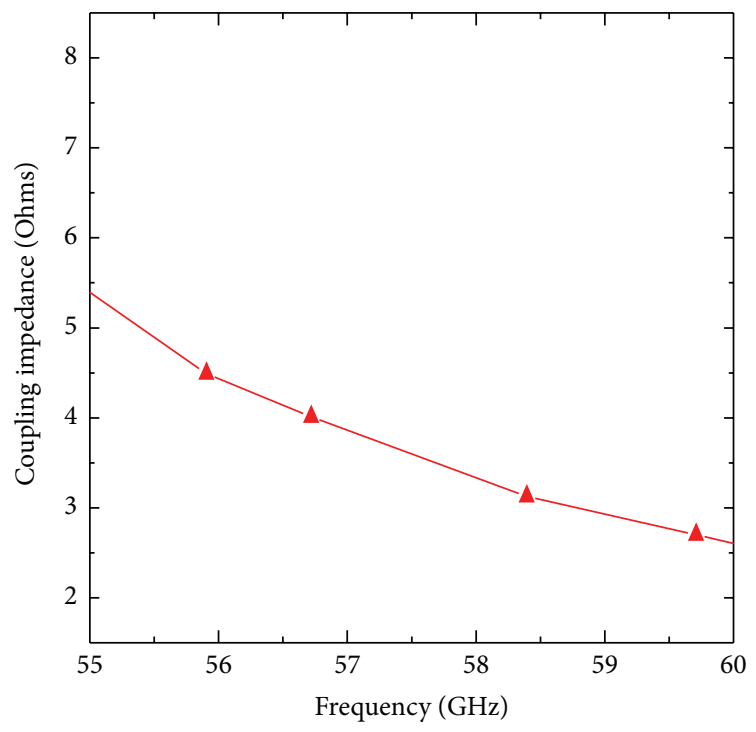

(b)

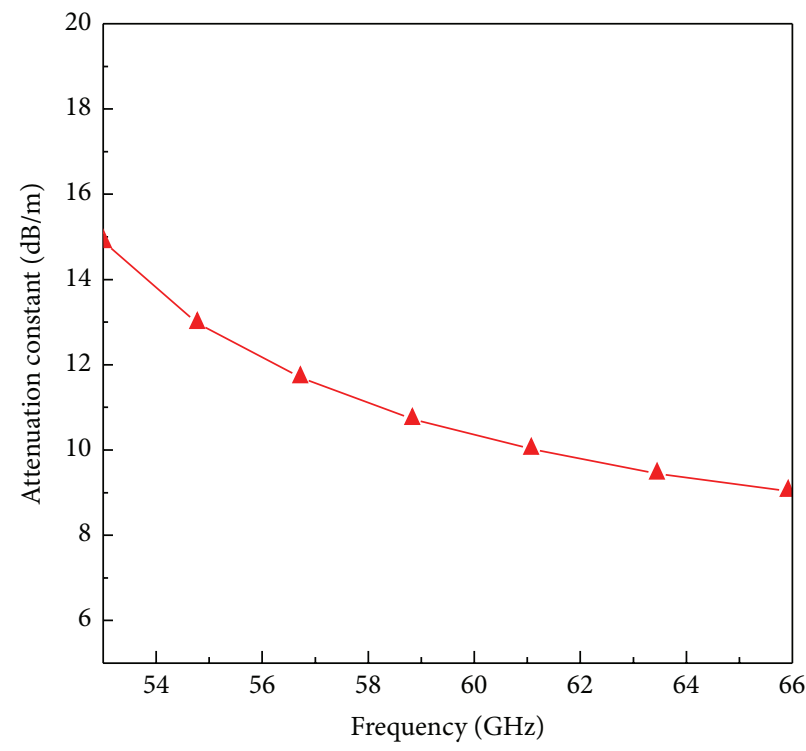

(c)

FIGURE 8: (a) Dispersion curve, (b) coupling impedance, and (c) attenuation constant for FWG SWS.

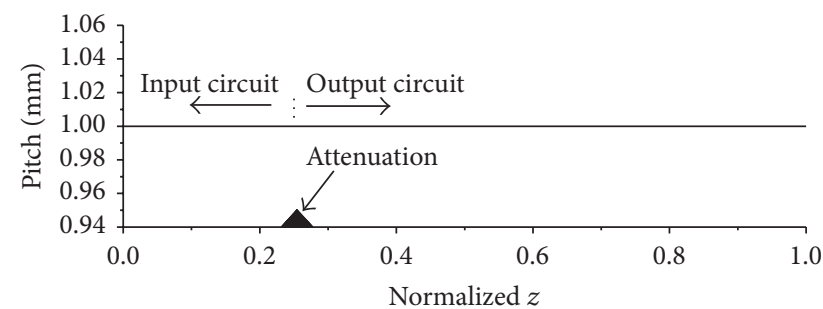

FIGURE 9: Pitch and attenuation profile of the FWG TWT (black and white).

stage helix TWT can achieve $34 \mathrm{~W}$ and the saturated gain of $35 \mathrm{~dB}$ from $55 \mathrm{GHz}$ to $60 \mathrm{GHz}$.
TABLE 1: Parameters of the helix slow-wave structure.

\begin{tabular}{lc}
\hline Parameter & Value \\
\hline Helix radius $\left(R_{a}\right)$ & $0.25 \mathrm{~mm}$ \\
Helix tape thickness & $0.08 \mathrm{~mm}$ \\
Helix tape width & $0.20 \mathrm{~mm}$ \\
Helix pitch & $0.45-0.55 \mathrm{~mm}$ \\
Rod material & APBN \\
Rode wedge angle $(\theta)$ & $30 \mathrm{deg}$ \\
Shield radius $\left(R_{c}\right)$ & $1.2 \mathrm{~mm}$ \\
\hline
\end{tabular}

4.3. Design of FWG TWT. Similar optimization is done for the FWG TWT (structure is shown in Figure 7). To get large 


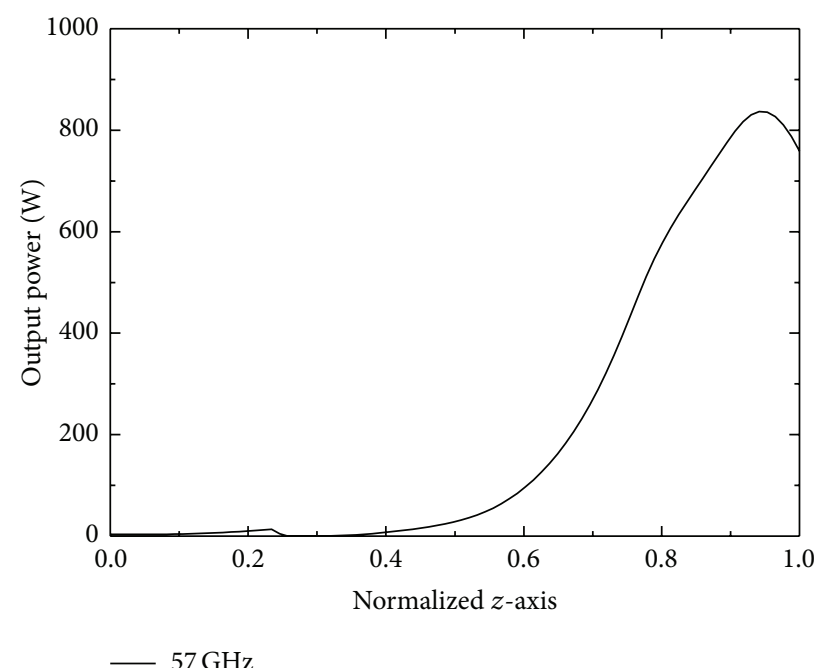

(a)

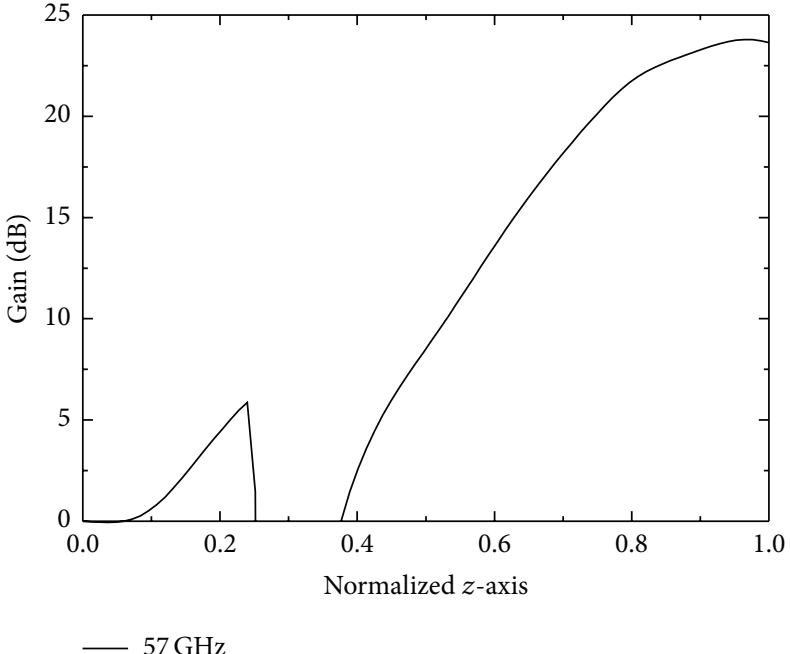

(b)

FIGURE 10: (a) Output power versus $z$ and (b) gain versus $z$ at $57 \mathrm{GHz}$ of the FWG TWT (black and white).

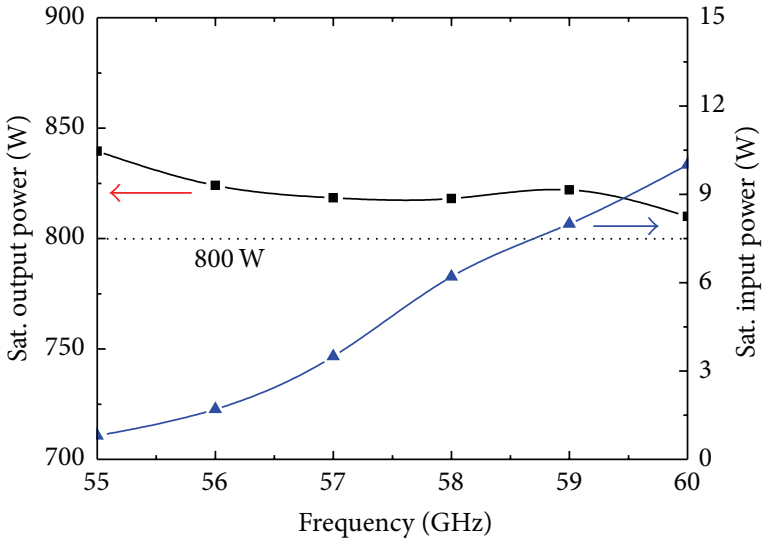

(a)

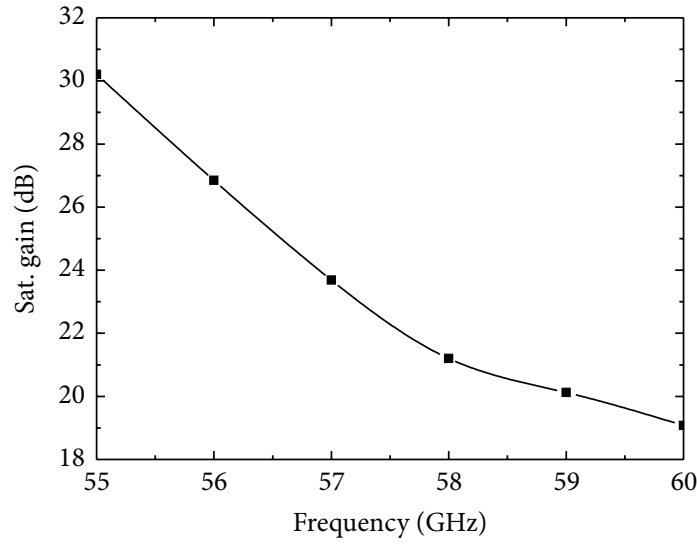

(b)

FIGURE 11: (a) Saturated output power and input power and (b) saturated gain of the FWG TWT ranging from $55 \mathrm{GHz}$ to $60 \mathrm{GHz}$.

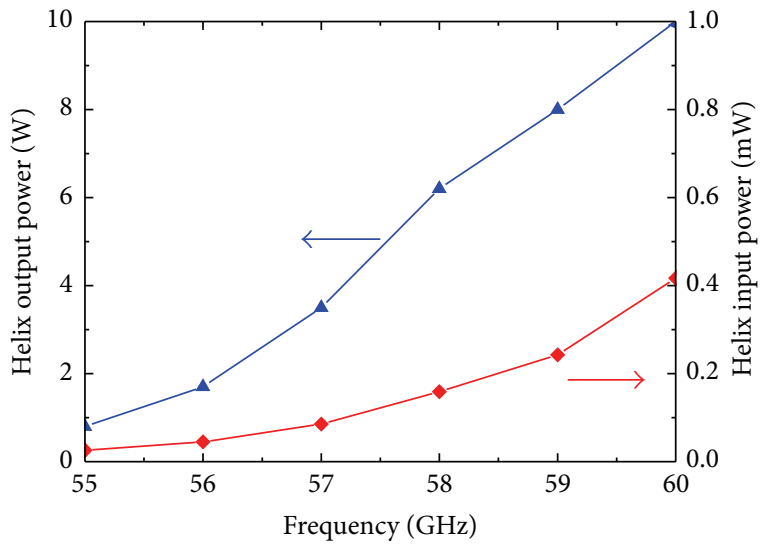

FIGURE 12: Helix output and input power curves to meet FW saturated input power.
TABLE 2: Parameters of the folded slow-wave structure.

\begin{tabular}{lc}
\hline Parameter & Value \\
\hline$r$ & $0.3 \mathrm{~mm}$ \\
$b$ & $3.15 \mathrm{~mm}$ \\
$a$ & $0.85 \mathrm{~mm}$ \\
$H$ & $0.8 \mathrm{~mm}$ \\
$L$ & $2.0 \mathrm{~mm}$ \\
\hline
\end{tabular}

coupling impedance and suitable synchronized velocity, we scan the structure parameters and finally get the FWG SWS with parameters listed in Table 2.

The normalized velocity, coupling impedance, and attenuation constant of the optimized FWG SWS are plotted in Figure 8. We can see that the relative discrepancy of normalized velocity is less than $1 \%$ from $55 \mathrm{GHz}$ to $60 \mathrm{GHz}$ in Figure 8(a), which indicates well dispersion flatness and 


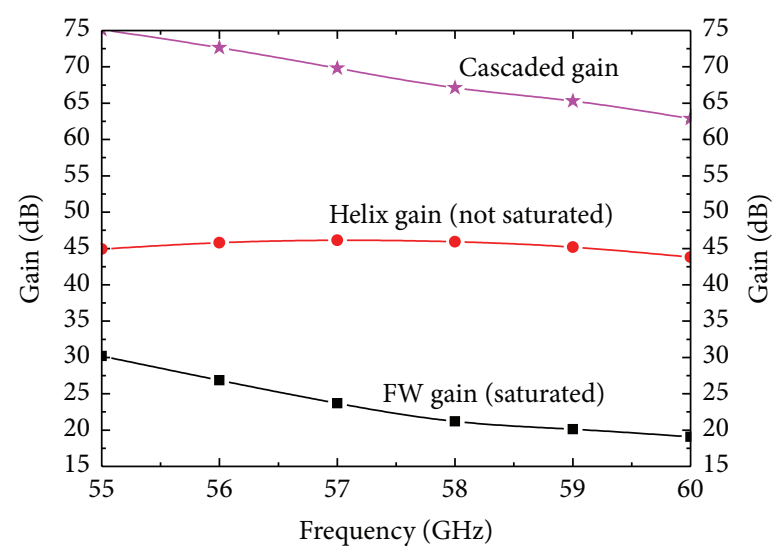

FIGURE 13: Cascaded gain, helix gain, and FW gain curves in band.

can satisfy the synchronization condition. As shown in Figure $8(\mathrm{~b})$, the coupling impedance is greater than $2.5 \mathrm{Ohms}$ within the designed V-band $55 \mathrm{GHz}$ to $60 \mathrm{GHz}$ and a strong beam-wave interaction can be expected. It can be found that the FWG SWS has much lower loss than helix one comparing Figures 8(c) and 4(c). Because it is the surface wave traveling along the helix with strong field in helix TWT, it is light field on the boundary of the waveguide in FWG SWS.

A large amount of optimization processes has been done to determine the profile of the FWG TWT and a high power pitch profile is proposed in Figure 9. A sever is select to cut the input circut short. Note that the pitch here refers to halfperiod of the FWG SWS.

The performance of the output power and gain at $57 \mathrm{GHz}$ are provided in Figure 10. And the saturated output power and the corresponding input power, as well as the saturated gain, are shown in Figure 11. Obviously, the saturated output power is greater than $800 \mathrm{~W}$ and the saturated gain is larger than $18 \mathrm{~dB}$ from $55 \mathrm{GHz}$ to $60 \mathrm{GHz}$.

After design of the first stage helix TWT and FWG TWT separately, we match the output power of helix TWT to be the same of the input power FWG TWT by modifying the helix input power, keeping the output power of FWG saturated (see Figure 12). Then, the saturated cascaded gain can be obtained by saturated FWG gain plus gain of helix TWT. As shown in Figure 13, the cascaded gain above $60 \mathrm{~dB}$ can be expected within the V-band of $55 \mathrm{GHz}$ to $60 \mathrm{GHz}$.

\section{Conclusions}

Based on the digitized nonlinear theory model developed in MTSS, a novel high power V-band cascaded helix-FWG TWT is designed, which is expected to generate an output power of $800 \mathrm{~W}$ and a gain of $60 \mathrm{~dB}$ from $55 \mathrm{GHz}$ to $60 \mathrm{GHz}$. The digitized nonlinear theory model, which can process the beam-wave interaction universally, is briefly introduced. And then the principle of the TWT design is derivate, by which a proper voltage and tunnel radius can be selected. The first stage helix traveling wave tube is designed to have an output power above $34 \mathrm{~W}$ and gain above $35 \mathrm{~dB}$. For the cascaded FWG traveling wave tube, the saturated output power is designed to be greater than $800 \mathrm{~W}$ and the saturated gain is larger than $18 \mathrm{~dB}$ in the $5 \mathrm{GHz}$ bandwidth. As a result, we get the expected output power of $800 \mathrm{~W}$ and gain of $60 \mathrm{~dB}$ by cascading these two traveling wave tubes.

\section{Conflict of Interests}

The authors declare that there is no conflict of interests regarding the publication of this paper.

\section{Acknowledgment}

This work was supported by the National Natural Science Foundation of China (Grant no. 61201003).

\section{References}

[1] G. K. Kornfeld, E. Bosch, W. Gerum, and G. Fleury, "60-GHz space TWT to address future market," IEEE Transactions on Electron Devices, vol. 48, no. 1, pp. 68-71, 2001.

[2] C. K. Chong and W. L. Menninger, "Latest advancements in high-power millimeter-wave helix TWTs," IEEE Transactions on Plasma Science, vol. 38, no. 6, pp. 1227-1238, 2010.

[3] A. Staprans, E. W. McCune, and J. A. Ruetz, "High power linearbeam tubes," Proceedings of the IEEE, vol. 61, no. 3, pp. 299-330, 1973.

[4] L. Li, J. J. Feng, B. Qu, and Y. H. Shang, "Design and experiment of a V-band helix TWT," in Proceedings of the IEEE 14th International Vacuum Electronics Conference (IVEC'13), pp. 1-2, Paris, France, 2013.

[5] L. Liu, Y. Wei, F. Shen et al., "A novel winding microstrip meander-line slow-wave structure for V-band TWT," IEEE Electron Device Letters, vol. 34, no. 10, pp. 1325-1327, 2013.

[6] F. Shen, Y. Y. Wei, H. R. Yin et al., "A novel V-shaped microstrip meander-line slow-wave structure for W-band MMPM," IEEE Transactions on Plasma Science, vol. 40, no. 2, pp. 463-469, 2012.

[7] Y. Gong, W. Wang, Y. Wei, and S. Liu, "Theoretical analysis of ridge-loaded ring-plane slow wave structure by variational methods," IEE Proceedings-Microwaves, Antennas and Propagation, vol. 145, no. 5, pp. 397-405, 1998.

[8] J. R. Legarra, J. Cusick, R. Begum, P. Kolda, and M. Cascone, "A 500-W coupled-cavity TWT for Ka-band communication," IEEE Transactions on Electron Devices, vol. 52, no. 5, pp. 665668, 2005.

[9] S. Liu, "Folded waveguide circuit for broadband MM wave TWTs," International Journal of Infrared and Millimeter Waves, vol. 16, no. 4, pp. 809-815, 1995.

[10] H. Gong, J. Xu, T. Tang et al., "A 1-kW 32-34-GHz folded waveguide traveling wave tube," IEEE Transactions on Plasma Science, vol. 42, no. 1, pp. 8-12, 2014.

[11] K. Nguyen, D. K. Abe, L. Ludeking et al., "High-power broadband cascaded-TWT development," in Proceedings of the IEEE 13th International Vacuum Electronics Conference (IVEC '12), pp. 121-122, Monterey, Calif, USA, April 2012.

[12] K. Nguyen, D. Pershing, J. Pasour et al., "Development of highpower broadband Ka-band cascaded-TWT," in Proceedings of the 14th IEEE International Vacuum Electronics Conference (IVEC '13), Paris, France, May 2013.

[13] B. Levush, D. Abe, I. A. Chernyavskiy et al., "A $1.8 \mathrm{~kW}$ broad band Ka-band TWT power booster," in Proceedings of the 14th 
IEEE International Vacuum Electronics Conference (IVEC '13), pp. 1-2, IEEE, Paris, France, May 2013.

[14] W. F. Peng, Y. L. Hu, Z. Cao, and Z. H. Yang, "Digitized nonlinear beam and wave interaction theory of traveling wave tube amplifiers," Progress In Electromagnetics Research M, vol. 28, pp. 73-88, 2013.

[15] W. F. Peng, Z. H. Yang, Y. L. Hu et al., “Time-dependent nonlinear theory and numerical simulation of folded waveguide traveling wave tubes," Journal of the Korean Physical Society, vol. 62, no. 6, pp. 949-953, 2013.

[16] B. Li, J.-Q. Li, Q. Hu et al., "Recent developments to the microwave tube simulator suite," IEEE Transactions on Electron Devices, vol. 61, no. 6, pp. 1735-1741, 2014.

[17] Ansoft Corporation, Ansoft HFSS User's Reference, Ansoft Corporation, 2015, http://www.ansoft.com.cn/.

[18] CST Microwave Studio, 3-D Electromagnetic Simulation Software, CST Corporation, Darmstadt, Germany, 2015. 

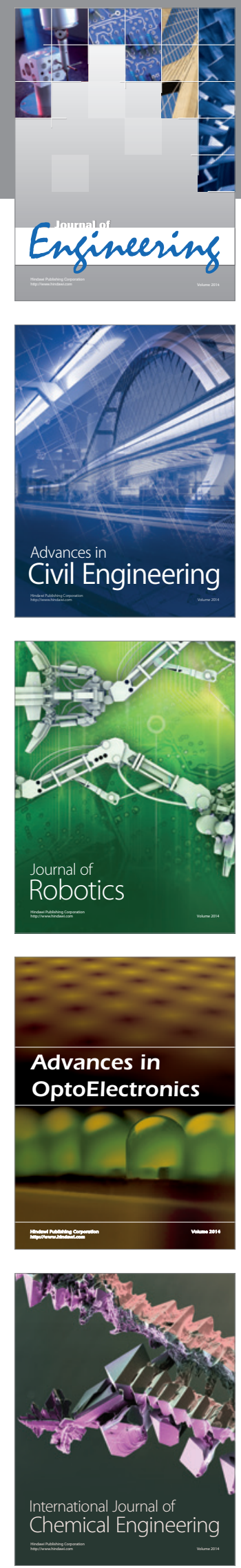

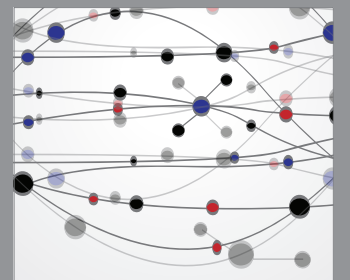

The Scientific World Journal
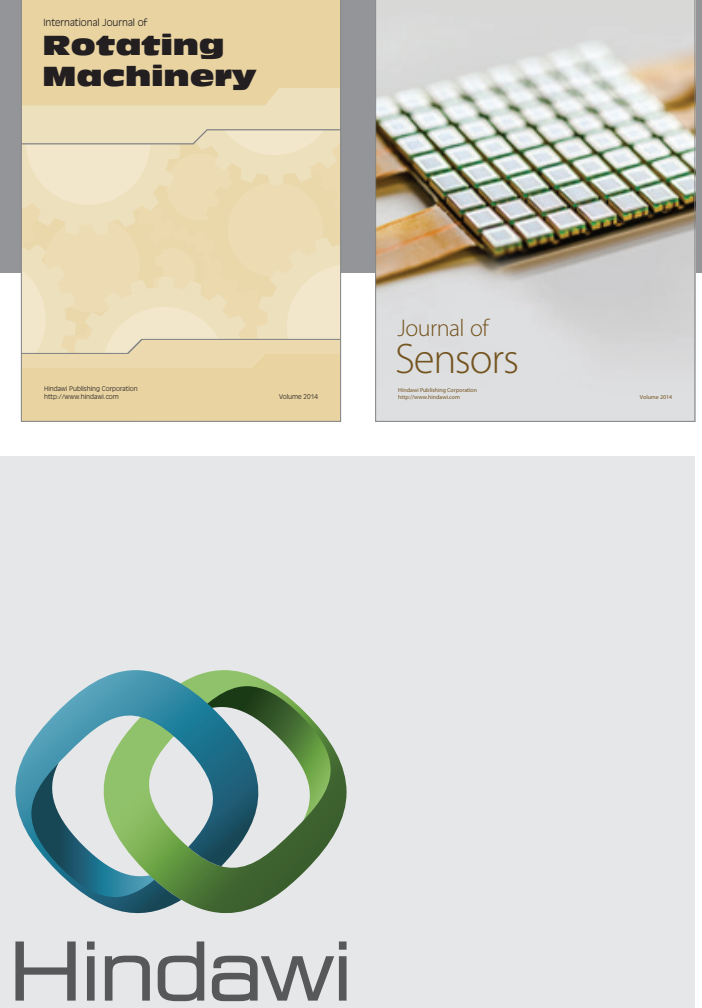

Submit your manuscripts at http://www.hindawi.com
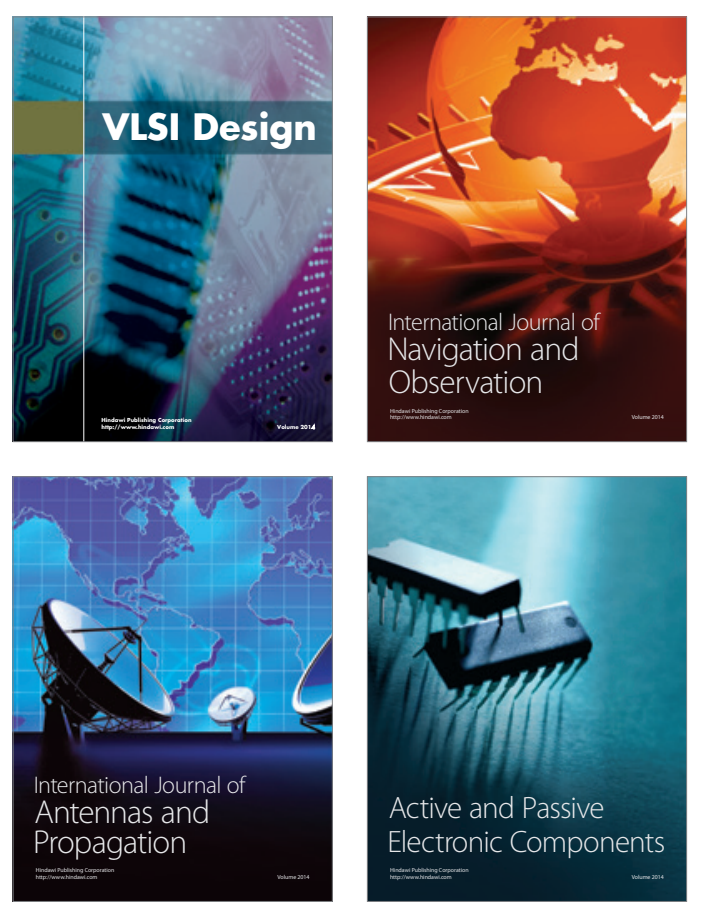
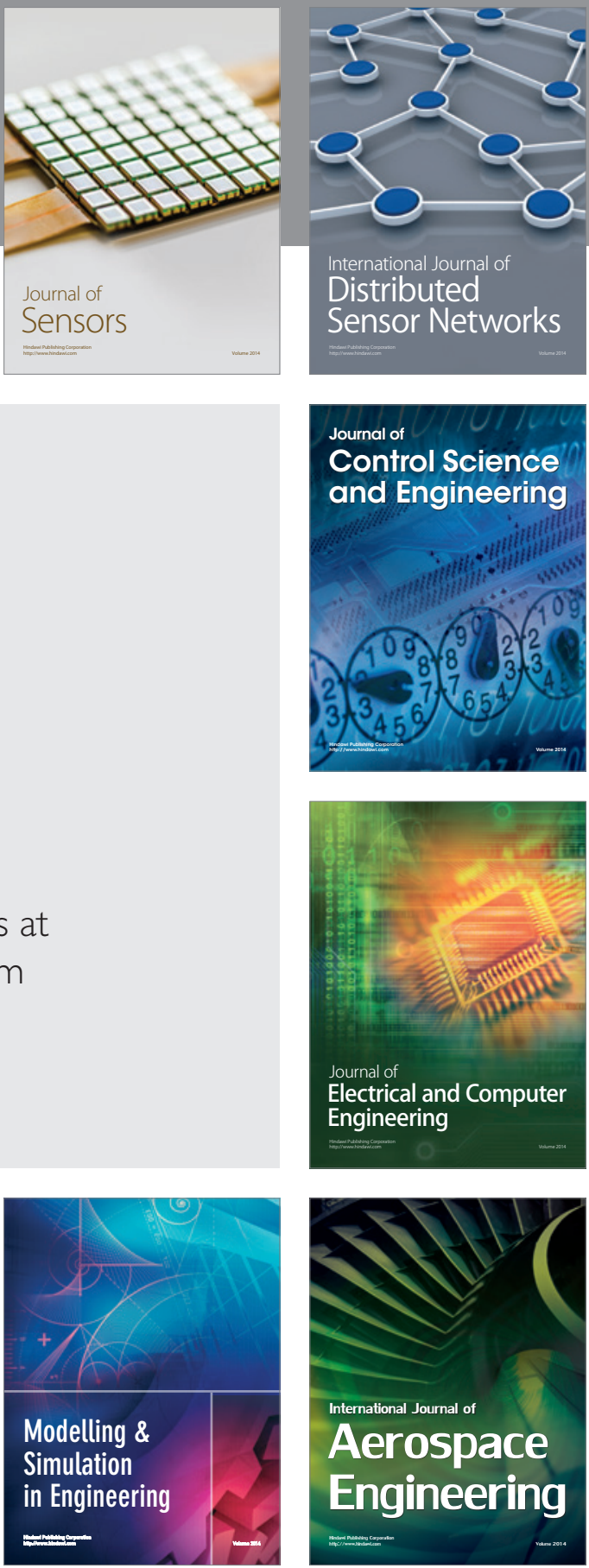

Journal of

Control Science

and Engineering
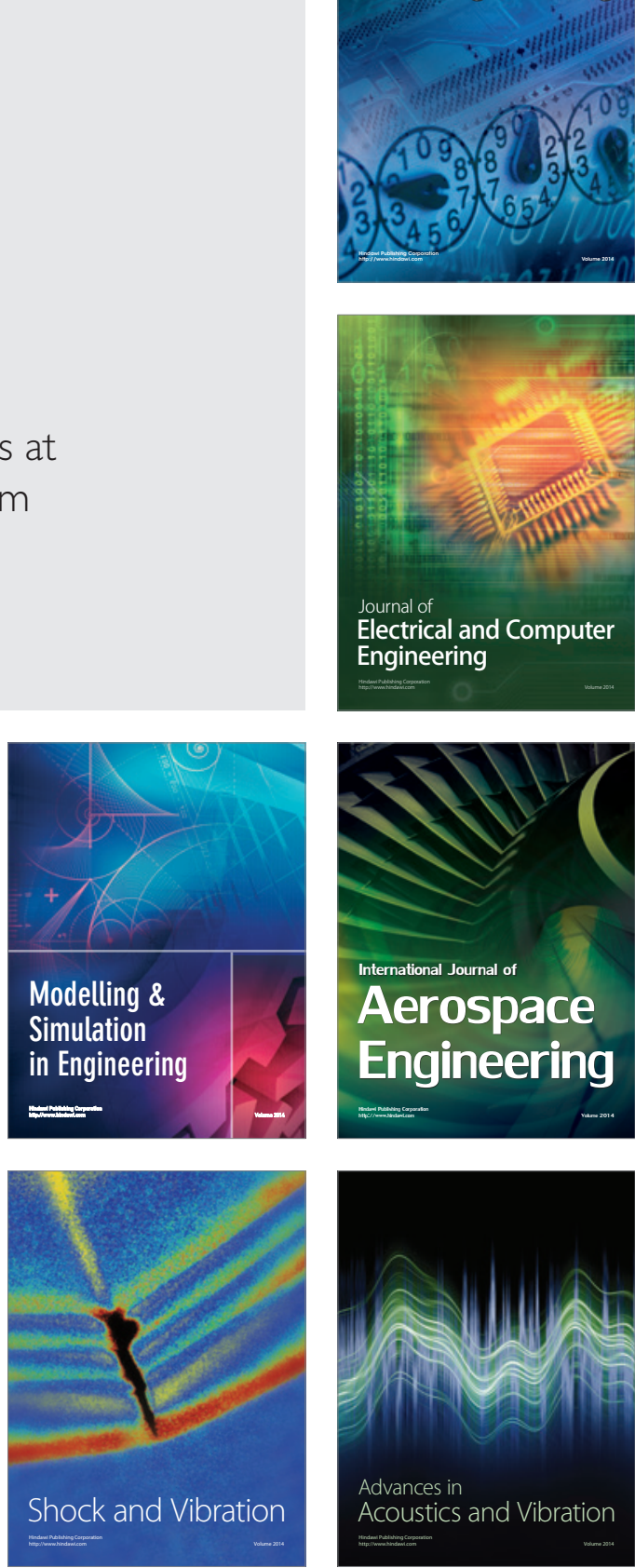